\title{
Repetitive Model Predictive Approach to Individual Pitch Control of Wind Turbines
}

\author{
Johannes Friis, Ebbe Nielsen, Jesper Bonding, Fabiano Daher Adegas, Jakob Stoustrup, Peter Fogh Odgaard
}

\begin{abstract}
A novel model predictive (MPC) approach for individual pitch control of wind turbines is proposed in this paper. A repetitive wind disturbance model is incorporated into the MPC prediction. As a consequence, individual pitch feedforward control action is generated by the controller, taking "future" wind disturbance into account. Information about the estimated wind spatial distribution one blade experience can be used in the prediction model to better control the next passing blade. A simulation comparison between the proposed controller and an industry-standard PID controller shows better mitigation of drive-train, blade and tower loads.
\end{abstract}

\section{INTRODUCTION}

The viability of energy sources are closely connected to the cost of energy (COE) generated. Historically, the natural way to achieve better COE of wind energy sources is to increase the size of wind turbines. A drawback of larger wind turbine rotors is the significant differences in wind speeds over the rotor plane, leading to unwanted asymmetrical structural loads, which could in turn shorten the lifetime of the different mechanical components. Active load mitigation by the wind turbine controller is often used to prevent the unwanted vibrations [1]. Most solutions adopted by the wind industry revolve around collective or cyclic pitch using classic control techniques. Individual pitch control has been studied [2] and first field tests were recently reported [3] to strength the viability of such control philosophy.

Modern controllers have also been researched throughout the past decade, e.g. $L Q R, H_{\infty}, H_{2}$. More recently, model predictive control (MPC) of wind turbines is an active research topic showing promising simulation results [4], [5], [6]. An uniform wind field equally distributed over the rotor plane is usually considered for MPC design purposes. Wind turbines are inherently exposed to non-uniform wind fields composed of wind shear, tower shadow, and possible wake contributions. Asymmetrical aerodynamic rotor loads are a consequence of such periodic, repetitive wind disturbances experienced by the blades. A controller may estimate and use this peculiar disturbance pattern to better attenuate loads and regulate power by controlling the blade pitch angles individually. To the modest knowledge of the authors, none of the MPC schemes found on the literature explore the particular repetitive nature of the wind disturbances in order

The fourth author was supported by Project CASED - Concurrent Aeroservoelastic Analysis and Design of Wind Turbines. Fabiano D. Adegas and Jakob Stoustrup are with the Dept. of Electronic Systems, Aalborg University, 9220-DK Aalborg, Denmark. fda@es.aau.dk. Johannes Friis, Ebbe Nielsen, Jesper Bonding were M.Sc. students of the same Department. Peter Fogh Odgaard is with K.K. Electronics A/S, DK-7430 Ikast, Denmark. peodg@kk-electronic.com to better attenuate loads and regulate power by controlling the blade pitch angles individually.

This paper proposes a MPC strategy for individual pitch control of wind turbines. The assumption that wind disturbances experienced by the blades are quasi-static within a one third rotor revolution is utilized by the MPC to predict future trajectories of the plant. Information about the wind spatial distribution one blade experience is shifted in time and utilized to better control the next passing blade. A repetitive wind disturbance model is incorporated into the MPC predictor and thus feed-forward control action is generated taking "future" disturbances into account. A filtering scheme to estimate states and wind disturbances, based on a Kalman filter and blade root strain gauge measurements, is also proposed. A linearized wind turbine model suitable for computing the predicted plant trajectories is developed, including wind model, aerodynamics, kinematic models of fore-aft tower and blade flap displacements, drive-train and pitch system. Model parameters are tuned using system identification to match the performance of the aeroelastic code FAST. Simulation results with a non-linear wind turbine model of the MPC strategy and an industry-standard PID controller shows much superior mitigation of structural vibrations and equivalent load damages by the proposed controller.

\section{WIND TURBINE MODEL}

A wind turbine model suitable for computing the predicted plant trajectories is briefly presented. More details on the model derivation are given in [7].

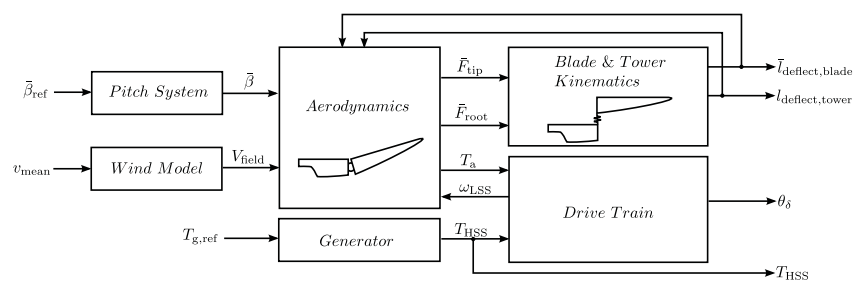

Fig. 1. Overall block diagram of the wind turbine model.

A block diagram of the wind turbine model structure is depicted on Fig. 1, where the main model variables are presented: $v_{\text {mean }}$ is the mean wind at the hub height from which a wind field $V_{\text {field }}$ over the rotor plane is constructed, $\bar{\beta}_{\text {ref }}$ and $\bar{\beta}$ are vectors with reference and actual blade pitch angles respectively, $T_{a}$ is the aerodynamic torque produced by the rotor, $\bar{F}_{\text {root }}$ and $\bar{F}_{t i p}$ is the thrust force contribution of the root part and the tip part of the blades, $\bar{l}_{\text {deflect, blade }}$ 
and $\bar{l}_{\text {deflect,tower }}$ are the blade and tower deflection lengths, respectively, $\omega_{L S S}$ and $\theta_{\delta}$ are the angular velocity and torsional angle of the low speed shaft, respectively.

\section{A. Wind Model}

The wind model generates, from a scalar mean wind speed at hub height, a time-varying matrix that contains the wind speed for each point in the wind field,

$$
\begin{aligned}
V_{\text {field }}(t, r, \theta)=v_{\text {mean }}(t)+V_{w s}(t, r, \theta) & +V_{t s}(t, r, \theta) \\
& +V_{w k}(t, r, \theta)
\end{aligned}
$$

where $V_{\text {field }}$ is the total wind speed field, $v_{\text {mean }}$ is the mean wind speed, $V_{w s}$ is the wind shear component, $V_{t s}$ is the tower shadow component, and $V_{w k}$ is the far wake component of one preceding wind turbine. Notice the dependence on the rotor radius $r$ and rotor azimuth angle $\theta$. Wind shear and tower shadow components were modeled as [8] and expressions for such will be omitted for brevity. The simplified wake model is represented as a part of the wind field (i.e. a circle) with a lower wind speed. The wake is centered around a point $\left(r_{0}, \varphi\right)$ placed on the rim of the wind field (Fig. 2(b)) and described mathematically as (2),

$$
r^{2}-2 r r_{0} \cos (\theta-\varphi)+r_{0}^{2}=a^{2}
$$

where $r_{0}$ is the radial coordinate for the center of the wake, $\varphi$ is the angular coordinate of the center of the wake and $a$ is the radius of the wake.

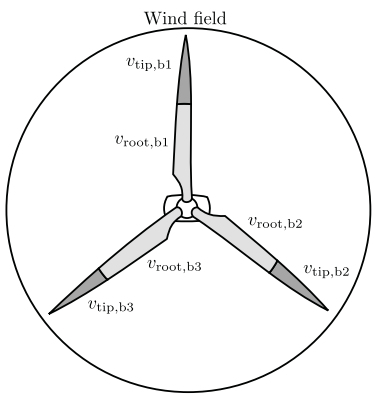

(a) Root and tip segments

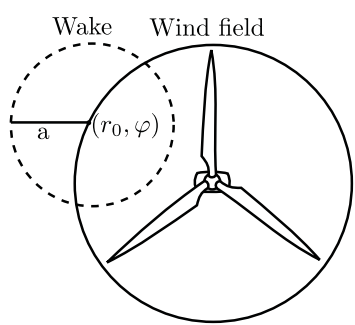

(b) Wake placement.
Fig. 2. Some wind field definitions.

Stochastic variables are add to the wind components except tower shadow, giving a closer to reality parameterization of the wind speeds throughout the rotor plane. Wind shear is based on the typical exponential law with coefficient $\alpha_{w s}$ chosen to be modelled as a nomally distributed random variable with mean and variance 0.194 and 0.137 , respectively [9]. The stochastic elements of the wake are the angular coordinate of wake center with variance 0.01 , and radius of the wake with variance 0.3 , both with zero mean. The wind field for a particular time stamp is illustrated on Fig. 3.

The infinite-dimensional wind field is converted to six equivalent winds signals that acts on two distinct parts of the blades, namely tip and root sections (Fig. 2(b)), in order

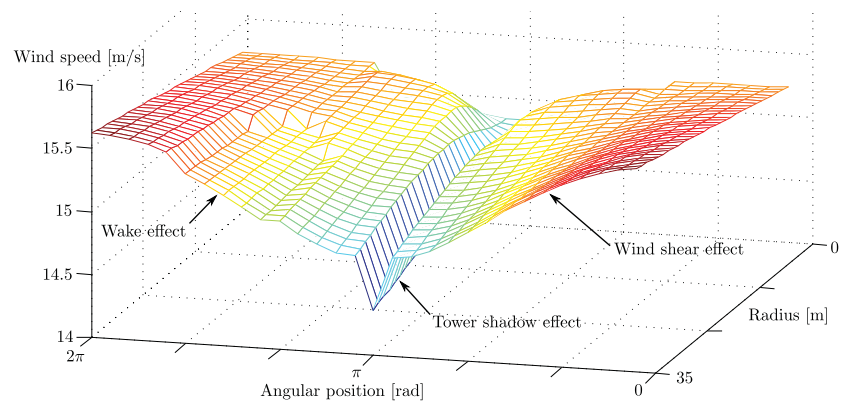

Fig. 3. Wind field containing wake, tower shadow and wind shear effects, for a particular time stamp.

to turn a linearized model viable,

$$
v_{\text {root }, b_{i}}=\sum_{r=0}^{r_{H}} v\left(r, \theta_{b_{i}}\right), \quad v_{t i p, b_{i}}=\sum_{r=r_{H}}^{R} v\left(r, \theta_{b_{i}}\right)
$$

where $r_{H}$ is the length from the hub to the hinge of the blade (shown in the sequel), $R$ is the length of the blade and $\theta_{b_{i}}$ is the azimuth angle of the ith blade.

\section{B. Aerodymamic Model}

The blade is modeled as two rigid parts (root and tip) connected with a hinge. The relevant forces and torques are depicted on Fig. 4(a): the low speed shaft torque $T_{L S S}$; the axial and tangential forces for both root and tip part of the blade, $F_{\text {root }, a}, F_{\text {root }, t}$ and $F_{t i p, a}, F_{t i p, t}$ respectively.

Aerodynamic forces and torques are computed by Blade Element Theory. The total force acting on the entire blade is the sum of forces acting on the blade elements. The blade geometry is approximated by three subparts as shown in Fig. 4(b) and is able to flap around the $r_{H}$ position. The axial forces produced by the root $\left(F_{\text {root }_{a}}\right)$ and tip $\left(F_{\text {tipa }}\right)$ are given by,

$$
\left[\begin{array}{c}
F_{r_{\text {oot }}}^{\prime} \\
F_{\text {tip }}^{\prime}
\end{array}\right]=\left[\begin{array}{c}
\frac{\rho}{2}\left(\sum_{i=0}^{i_{r_{W}}} V_{e f f}^{2}\left(r_{i}, \theta\right) f_{b l 1}\left(r_{i}\right) \Delta r\right. \\
\left.+\sum_{i=i_{r_{W}}}^{i_{r_{H}}} V_{e f f}^{2}\left(r_{i}, \theta\right) f_{b l 2}\left(r_{i}\right) \Delta r\right) \\
\frac{\rho}{2}\left(\sum_{i=i_{r_{H}}}^{i_{R}} V_{e f f}^{\prime}{ }_{\text {ef }}^{2}\left(r_{i}, \theta\right) f_{b l 2}\left(r_{i}\right) \Delta r\right)
\end{array}\right]
$$

The airfoil local angle of attack $\alpha$, local inflow angle $\psi$ and local inflow velocities $V_{\text {eff }}$ and $V_{\text {eff }}^{\prime}$ are given by,

$$
\begin{aligned}
\alpha= & \tan ^{-1}\left(\frac{V_{\text {field }}-\dot{x}_{t}-\dot{x}_{b}}{\omega_{L S S} r}\right)-\left(\beta+\beta_{\text {twist }}\right) \\
\psi= & \tan ^{-1}\left(\frac{V_{\text {field }}-\dot{x}_{t}-\dot{x}_{b}}{\omega_{L S S} r}\right) \\
V_{\text {eff }}= & \sqrt{\left(V_{\text {field }}-\dot{x}_{t}-\dot{x}_{b}\right)^{2}+\left(\omega_{L S S} r\right)^{2}}, \forall r_{i} \leq r_{H} \\
V_{\text {eff }}^{\prime}= & \sqrt{\left(\cos \left(\theta_{\text {hg }}\right)\left(V_{\text {field }}-\dot{x}_{t}-\dot{x}_{b}\right)\right)^{2}+\left(\omega_{L S S} r\right)^{2}}, \\
& \forall r_{i}>r_{H}
\end{aligned}
$$


where $C_{L}(\alpha)$ is the airfoil lift force coefficient, $C_{D}(\alpha)$ is the airfoil drag force coefficient, $\rho$ is the air density, $\Delta r$ is the length of the blade element, $f_{b l 1}\left(r_{i}\right)$ is the function that describes first interval and $f_{b l 2}\left(r_{i}\right)$ the second and third interval areas of the blade. Projecting $F_{t i p_{a}}^{\prime}$ on the axial axis and summing the forces for all three blade intervals results in the total thrust force generated by one blade,

$$
F_{x}=F_{\text {root }_{a}}+\cos \left(\theta_{\text {hinge }}\right) F_{\text {tipa }_{a}}^{\prime} .
$$

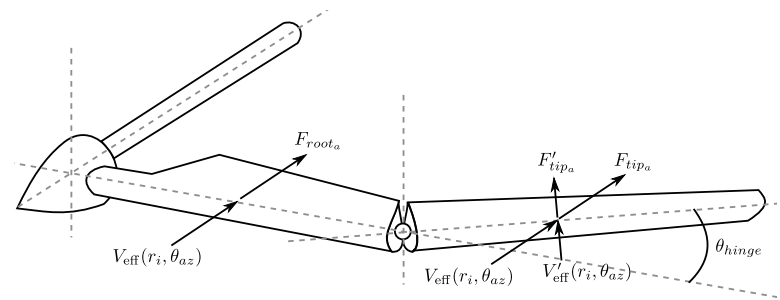

(a) Hinged blade with relevant forces and torques.
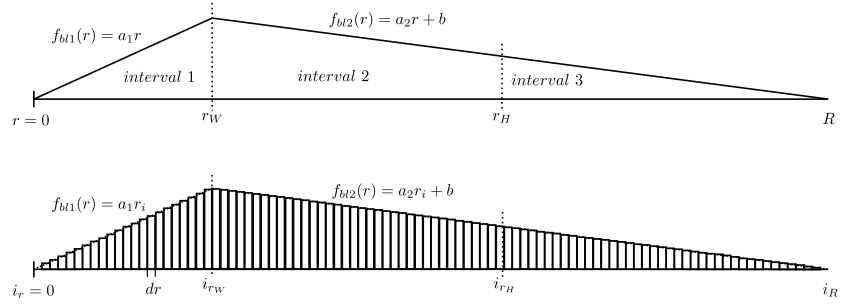

(b) Approximated geometry and discretization by blade elements.

Fig. 4. Aerodynamic definitions.

For the aerodynamic torque, tangential forces acting on the blade element must be multiplied with the distance to the shaft center, resulting in the following description of torque produced by the tip and root segments,

$$
\left[\begin{array}{c}
T_{\text {root }} \\
T_{\text {tip }}
\end{array}\right]=\left[\begin{array}{c}
\frac{\rho}{2}\left(\sum_{r=0}^{i_{r_{W}}} V_{e f f}^{2}\left(r_{i}, \theta\right) f_{b l 1}\left(r_{i}\right) \Delta r\right. \\
\left.+\sum_{r=i_{r_{W}}}^{i_{r_{H}}} V_{e f f}^{2}\left(r_{i}, \theta\right) f_{b l 2}\left(r_{i}\right) \Delta r\right) \\
\frac{\rho}{2}\left(\sum_{r=i_{r_{H}}}^{i_{R}} V_{e f f}^{\prime 2}\left(r_{i}, \theta\right) \Delta r l_{h, i}\right) \\
\cdot\left(C_{L}(\alpha) \sin (\psi)-C_{D}(\alpha) \cos (\psi)\right)
\end{array}\right]
$$

where

$$
\begin{aligned}
\theta_{\text {hinge }} & =\sin ^{-1}\left(\frac{l_{\text {deflect }, \text { blade }, i}}{l_{\text {tip }}}\right) \\
l_{t, i} & =\cos \left(\theta_{\text {hinge }}\right) l_{\text {tip }} \\
l_{h, i} & =l_{\text {root }}+l_{t, i}
\end{aligned}
$$

and $l_{\text {deflect }, \text { blade, } i}$ comes from the kinematic model presented next. The total torque produced by a blade is then

$$
T_{a}=T_{\text {tip }}+T_{\text {root }}
$$

\section{Kinematics}

The fore-aft tower and flap blade deflections are modeled as multiple connected mass-spring-damper systems. The model consists of three masses for the blade tips, parallel connected to the tower/blade root system. Equations of motion for the i-th blade and tower are

$$
\begin{aligned}
\ddot{x}_{b_{i}} & =\frac{F_{t i p, b_{i}}}{m_{b_{i}}}+\frac{K_{b_{i}}}{m_{b_{i}}} x_{t}-\frac{K_{b_{i}}}{m_{b_{i}}} x_{b_{i}}+\frac{B_{b_{i}}}{m_{b_{i}}} \dot{x}_{t}-\frac{B_{b_{i}}}{m_{b_{i}}} \dot{x}_{b_{i}} \\
\ddot{x}_{t} & =\sum_{i=1}^{3}\left(\frac{K_{b_{i}}}{m_{b_{i}}} x_{b_{i}}+\frac{B_{b_{i}}}{m_{b_{i}}}(x)_{b_{i}}+\frac{F_{t i p, b_{i}}}{m_{t}}+\frac{F_{\text {root }, b_{i}}}{m_{t}}\right) \\
& -\frac{1}{m_{t}}\left(\left(K_{b_{1}}+K_{b_{2}}+K_{b_{3}}+K_{t}\right) x_{t}\right. \\
& \left.+\left(B_{b_{1}}+B_{b_{2}}+B_{b_{3}}+B_{t}\right) \dot{x}_{t}\right) .
\end{aligned}
$$

It shall be noted that the mass $m_{t}$ consists both of the tower mass and the root masses of the three blades. The output deflections are defined as,

$$
\begin{aligned}
l_{\text {deflect }, \text { blade }, i} & =x_{b_{i}}-x_{t} \\
l_{\text {deflect }, \text { tower }} & =x_{t}
\end{aligned}
$$

Drive-train is modeled as a inertial two-mass system with an ideal planetary gear and torsional spring referenced to the low speed shaft,

$$
\begin{aligned}
J_{r} \dot{\omega}_{L S S}(t)=T_{a}(t)-K_{d t} \theta_{\delta}(t)-\left(B_{d t}+B_{r}\right) \omega_{L S S}(t) \\
+\frac{B_{d t}}{N_{g}} \omega_{H S S}(t) \\
J_{g} \dot{\omega}_{H S S}(t)=\frac{K_{d t}}{N_{g}} \theta_{\delta}(t)+\frac{B_{d t}}{N_{g}} \omega_{L S S}(t)-T_{g}(t) \\
-\left(\frac{B_{d t}}{N_{g}^{2}}+B_{g}\right) \omega_{H S S}(t) \\
\dot{\theta}_{\delta}(t)=\omega_{L S S}(t)-\frac{1}{N_{g}} \omega_{H S S}(t)
\end{aligned}
$$

where $\omega_{H S S}$ is the angular velocity on the high speed shaft, $J_{r}$ and $J_{g}$ are rotor and generator inertias, $K_{d t}$ and $B_{d t}$ are drive-train stiffness and damping coefficients, and $N_{g}$ is the gearbox ratio.

\section{Linearization}

The main aspects of the model linearization over a mean wind speed are briefly outlined. The blade twist, which is not uniform throughout the length of the blade, is averaged over the root $\left(\beta_{\text {twist,root }}\right)$ and tip $\left(\beta_{\text {twist,tip }}\right)$. The location where the tip and root wind speeds are applied on the blade span is based on the assumption that the total torque produced by the blade can be divided in two parts, one generated by the tip and another by the root. Therefore, the sum of wind speeds over the root/tip, when applied at only one point on the blade span, should generate approximately the same total torque and thrust. $C_{L}$ and $C_{D}$ curves are linearly approximated over the operating point. A linear approximation is justified because, when pitch control regulates generated power at rated, wind turbines operates most of the time on the linear region of $C_{L}$ and $C_{D}$ curves. 
The twist angle of the blades and the two span distances where the tip and root wind speeds are applied to the blade are utilized as a tuning parameters to fit the aerodynamic model to the response of the aeroelastic code FAST [10] subroutine AeroDyn. The model parameters were adjusted to resemble the $1.5 \mathrm{MW}$ reference wind turbine included in FAST [10]. Aerodynamic parameters were taken directly from respective data tables. Parameters for the kinematic model were obtained by grey-box system identification in a prediction error method scheme. The data utilized for identification were time-series simulations on FAST with step wind inputs. More details on the identification procedure are given in [7]. The resulting linear state-space system describing the wind turbine dynamics over a mean wind speed is given by,

$$
\dot{x}(t)=A x(t)+B_{s} u(t)+B_{d} d(t)
$$

where $x(t)$ is the state vector, $u(t)$ is the controlled inputs that includes generator torque and individual pitch angle signals, $d(t)$ is the unmeasured disturbance inputs composed of the tip and root wind speed signals for each blade

$$
\begin{aligned}
x(t)= & {\left[x_{b, 1}, \dot{x}_{b, 1}, x_{b, 2}, \dot{x}_{b, 2}, x_{b, 3}, \dot{x}_{b, 3}, x_{t}, \dot{x}_{t}, \omega_{L S S},\right.} \\
& \left.\omega_{H S S}, \theta_{\delta}, \beta_{1}, \beta_{2}, \beta_{3}\right]^{T} \\
u(t)= & {\left[T_{g}, \beta_{1, r e f}, \beta_{2, r e f}, \beta_{3, r e f}\right]^{T} } \\
d(t)= & {\left[v_{i n, t, 1}, v_{i n, t, 2}, v_{i n, t, 3}, v_{i n, r, 1}, v_{i n, r, 2}, v_{i n, r, 3}\right]^{T} . }
\end{aligned}
$$

The mean wind speed varies during wind turbine operation, thus a successive model linearization MPC scheme should be adopted in practice. This issue is not covered in the present paper.

\section{Repetitive Control Strategy}

The proposed repetitive control strategy is composed of a model predictive controller (MPC) with constraints, a repetitive wind disturbance model, and a state/disturbance estimator, depicted on Fig. 5. The unmeasurable states and wind disturbances are estimated in a Kalman Filter scheme by the Plant and Wind Estimator. The Memory \& Time Shifter stores and shifts forward on time the wind information estimated for a preceding blade in order to generate an estimate of the wind disturbances the next passing blade will experience. Together with the estimated states, is the basis for the predictor. The optimizer then calculates the optimal open-loop control signal $u(k)$ given the expected trajectories of the plant and wind disturbances.

The subcomponents of the proposed strategy are now explained in more details.

\section{A. Plant \& Wind Estimator}

Full state information and measured disturbances are required by the adopted MPC controller, thus estimates of unmeasurable states and wind disturbances are needed. Forces acting on the root and tip elements of the blade are estimated by a Kalman filter and later converted into wind speed signals by a linear coherence relation between states, forces, and wind disturbances. This particular estimation structure was

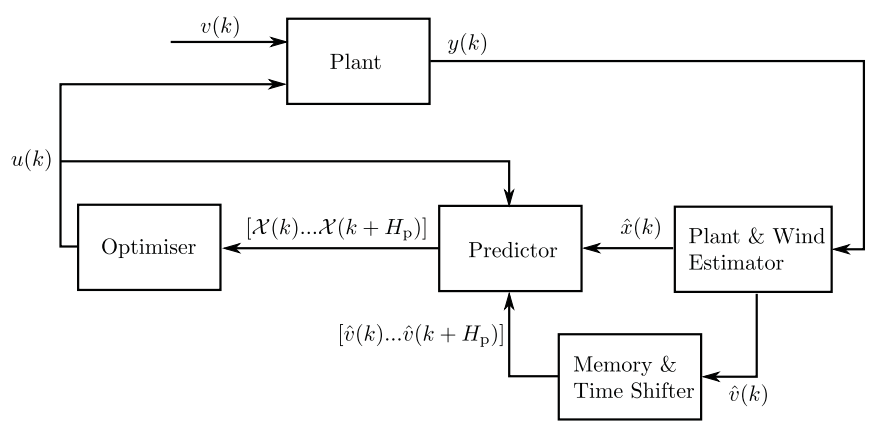

Fig. 5. Repetitive MPC control strategy for a wind turbine.

chosen to take advantage of strain gauge measurements of the combined force acting on a blade root, to improve the estimation. Thus, the system model used in the Kalman filter does not contain the linearized aerodynamic model, only the kinematic models with forces appearing explicitly into the formulation.

The force acting on each root and tip element can be estimated by the usage of the state error between plant and the Kalman filter. The Kalman filter has been augmented with six force states and one torque state modeled as first order low pass filters with cut-off frequency $f_{0}$. After a zero order hold discretization, the model used on the predict step of the Kalman filter is

$$
\begin{aligned}
\hat{x}^{-}(k) & =\Phi \hat{x}^{+}(k-1)+\Pi u(k-1) \\
\hat{z} & =H \hat{x}^{-}(k)
\end{aligned}
$$

where $\hat{x}^{-}$and $\hat{x}^{+}$are a priori and a posteriori estimate of the states, respectively, $\hat{z}$ is the estimate of the measured states. System matrices are,

$$
H=\left[\begin{array}{cc}
C_{k l m} & 0 \\
0 & I
\end{array}\right], \quad \Phi=\left[\begin{array}{cc}
A_{s} & B_{s} \\
0 & f_{0} I
\end{array}\right], \quad \Pi=\left[\begin{array}{c}
B_{s} \\
0
\end{array}\right]
$$

where $C_{k l m}$ selects blade and tower velocities $x_{b, i}, x_{t}$, shaft speeds $\omega_{L S S}, \omega_{H S S}$ and blade pitch angles $\beta_{i}$.

\section{B. Memory \& Time Shifter}

The Memory \& Time Shifter stores the estimates of the present wind speeds, shifts in time and sorts them to be adequately used by the MPC disturbance model. Figure 6 illustrates the subcomponents of the memory process. Their functionality and design criteria are described below.

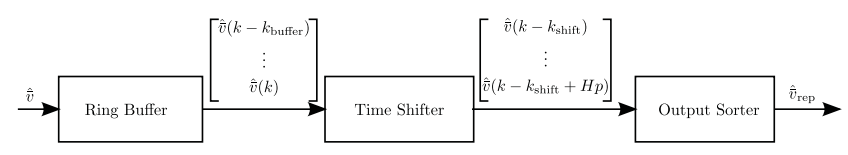

Fig. 6. Subcomponents of the Memory \& Time Shifter Block.

Ring Buffer: The size of the ring buffer is designed to the worst case scenario, corresponding to a full rotation at the slowest rotational speed. It is assumed that the rotational speed of the low speed shaft will not decrease below a 
minimum value on the operating range of interest. This yields a buffer size of

$$
k_{\text {buffer }}=\frac{2 \pi}{\omega_{L S S, \min }} \frac{1}{T_{s}}
$$

where $\omega_{L S S, \text { min }}$ is the worst case velocity of the low speed shaft.

Time Shifter: It determines the time shift needed to synchronize the stored buffer data with the present position of each blade. The value of $k_{\text {shift }}$ is calculated based on the velocity of the low speed shaft.

$$
k_{\text {shift }}=\frac{2 \pi \frac{b_{s}}{3}}{\omega_{L S S} T_{s}}
$$

where $b_{s}$ is the blade delay index. In order to secure causality, the blade delay index $b_{s}$ is adjusted runtime in order to ensure that

$$
k_{\text {shift }}>H_{p}
$$

Output Sorter: Before the horizon of wind speeds can be passed to the MPC the wind state vector $d_{m}(k)$ must be rearranged. This is done according to the blade delay index such that the buffered wind corresponds to the actual blade.

\section{Model Predictive Control with Contraints}

The model predictive controller and its governing equations are outlined here, heavily inspired on [11]. A Model Predictive Controller (MPC) is a model-based controller that, based on the current input and state values, uses an internal dynamic model of the plant to predict future system responses and compute an optimal finite-horizon open-loop control signal. Future responses are predicted over the horizon $\left(H_{p}\right)$ and are dependent on the assumed input trajectory $\hat{u}(k+i \mid k)$ applied over $\left(i=0,1, \ldots, H_{p}-1\right)$. The MPC manipulates the output $\hat{y}(k+i \mid k)$ towards some reference trajectory $r(k+i \mid k)$ by calculating an optimal finite-horizon open-loop input trajectory $u(k+i \mid k)$ over the control horizon $H_{u}$ The predicted input is assumed constant beyond $H_{u}$. Only the first sample from the predicted input is applied on the plant. A new input trajectory is computed at each $k$, implying that the horizon $H_{p}$ stays constant but shifted $k+1$, giving the next control signal plant input, setting $u(k+1)=\hat{u}(k+1 \mid k+1)$.

A feed-forward control action may be incorporated into the MPC if measured disturbances are included into the prediction model. In the proposed repetitive scheme, present and "future" values of wind disturbances, given by the Memory \& Time Shifter block, are utilized in the known disturbance part of the prediction model (stacked as $\mathcal{D}_{m}$ matrix shown ahead). The standard formulation of a quadratic MPC problem with measured disturbances, zero-offset tracking and linear matrix inequalities constraints is,

$$
\begin{gathered}
\text { Minimize } \Delta \mathcal{U}(k)^{T} \mathcal{H} \Delta \mathcal{U}(k)-\mathcal{G} \Delta \mathcal{U}(k) \\
\text { Subject to } \\
{\left[\begin{array}{c}
\mathcal{F} \\
\Gamma \Theta \\
W
\end{array}\right] \Delta \mathcal{U}(k) \leq\left[\begin{array}{c}
-\mathcal{F}_{1} u(k-1)-f \\
-\Gamma\left[\Psi \hat{x}(k)+\Upsilon u(k-1)+\Xi D_{m}(k)\right]-g \\
-w
\end{array}\right]}
\end{gathered}
$$

where

$$
\mathcal{H}=\Theta^{T} \mathcal{Q} \Theta+\mathcal{R}, \quad \mathcal{G}=2 \Theta^{T} \mathcal{Q} \mathcal{E}(k)
$$

The optimal assumed input trajectory resulted from the optimization problem is defined as,

$$
\Delta \mathcal{U}(k)=\left[\begin{array}{c}
\Delta \hat{u}(k \mid k) \\
\vdots \\
\Delta \hat{u}\left(k+H_{u}-1 \mid k\right)
\end{array}\right] .
$$

The terms in (21) are now detailed. The difference between the future target trajectory and the free system response, if no input changes were made, known as the "tracking error", is given by,

$$
\mathcal{E}(k)=\mathcal{T}(k+1)-\Psi x(k)-\Xi \mathcal{D}_{m}(k)-\Upsilon u(k-1) .
$$

$\mathcal{T}(k+1)$ is the horizon of references over $H_{p} . \mathcal{Q}, \mathcal{R}$ are the expansion of the weight matrices $Q$ and $R$ over $H_{p}$ and $H_{u}$, respectively,

$$
\begin{aligned}
& \mathcal{T}(k+1)=\left[\begin{array}{c}
\hat{r}(k+1 \mid r) \\
\vdots \\
\hat{r}\left(k+H_{p} \mid k\right)
\end{array}\right] \\
& \mathcal{Q}=\left[\begin{array}{cccc}
Q(1) & 0 & \ldots & 0 \\
0 & Q(2) & \ldots & 0 \\
\vdots & \vdots & \ddots & \vdots \\
0 & 0 & \ldots & Q\left(H_{p}\right)
\end{array}\right] \\
& \mathcal{R}=\left[\begin{array}{cccc}
R(0) & 0 & \ldots & 0 \\
0 & R(1) & \ldots & 0 \\
\vdots & \vdots & \ddots & \vdots \\
0 & 0 & \ldots & R\left(H_{u}\right)
\end{array}\right]
\end{aligned}
$$

The known input disturbance throughout the prediction horizon is stacked as $\mathcal{D}_{m}$. The $\Xi$ matrix defines the coupling of $\mathcal{D}_{m}$ to the dynamics of the prediction over $H_{p}$,

$$
\begin{gathered}
\mathcal{D}_{m}=\left[\begin{array}{c}
d_{m}(k) \\
\hat{d}_{m}(k+1 \mid k) \\
\vdots \\
\hat{d}_{m}\left(k+H_{p}-1 \mid k\right)
\end{array}\right] \\
\Xi=\left[\begin{array}{cccc}
B_{d} & 0 & \ldots & 0 \\
A B_{d} & B_{d} & \ldots & 0 \\
\vdots & \vdots & \ddots & \vdots \\
A^{H_{p}-1} B_{d} & A^{H_{p}-2} B_{d} & \ldots & B_{d}
\end{array}\right] .
\end{gathered}
$$

The matrix $\Psi$ incorporates the present state value and $\Upsilon$ and $\Theta$ couples the controlled inputs into the prediction model,

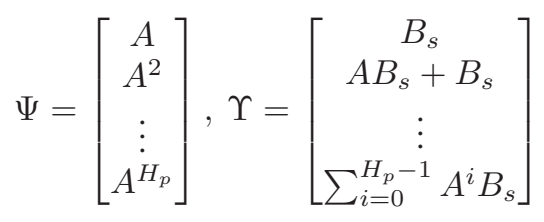


$\Theta=\left[\begin{array}{cccc}B_{s} & 0 & \ldots & 0 \\ A B_{s}+B_{s} & B_{s} & \ldots & 0 \\ \vdots & A B_{s}+B_{s} & \ddots & 0 \\ \vdots & \vdots & \ddots & \vdots \\ \sum_{i=0}^{H_{u}-1} A^{i} B_{s} & \sum_{i=0}^{H_{u}-2} A^{i} B_{s} & \ldots & B_{s} \\ \sum_{i=0}^{H_{u}} A^{i} B_{s} & \sum_{i=0}^{H_{u}-1} A^{i} B_{s} & \ldots & A B_{s}+B_{s} \\ \vdots & \vdots & \vdots & \vdots \\ \sum_{i=0}^{H_{p}-1} A^{i} B_{s} & \sum_{i=0}^{H_{p}-1} A^{i} B_{s} & \ldots & \sum_{i=0}^{H_{p}-H_{u}} A^{i} B_{s}\end{array}\right]$

Now the LMI constraint (20b) are detailed to clarify the missing parts of the formulation. Constraints can be attributed to predicted control input increments, control input and states,

$$
E\left[\begin{array}{c}
\Delta \mathcal{U}(k) \\
1
\end{array}\right] \leq 0, \quad F\left[\begin{array}{c}
\mathcal{U}(k) \\
1
\end{array}\right] \leq 0, \quad G\left[\begin{array}{c}
\mathcal{X}(k) \\
1
\end{array}\right] \leq 0
$$

where $\Delta \mathcal{U}(k)=\left[\hat{u}(k \mid k)^{T} \ldots \hat{u}\left(k+H_{u}-1 \mid k\right)^{T}\right]^{T}$ is defined analogously to $\mathcal{U}(k)$. As $F$ has the form $F=$ $\left[F_{1} F_{2} \ldots F_{H_{u}}, f\right]$ and defining $\mathcal{F}_{i}=\sum_{j=1}^{H_{u}} F_{j}, \mathcal{F}=$ $\left[\begin{array}{lll}\mathcal{F}_{1} & \ldots & \mathcal{F}_{H_{u}}\end{array}\right]$ the constraint matrix $F$ can be rewritten as the linear inequality,

$$
\mathcal{F} \Delta \mathcal{U}(k) \leq-\mathcal{F}_{1} u(k-1)-f
$$

that appears as the first inequality in (20b). The constraints on the predicted states $\mathcal{X}(k)$ can be put as the second inequality by letting $G=\left[\begin{array}{ll}\Gamma & g\end{array}\right]$ where $\mathrm{g}$ is the last column of $G$. At last, the constrains on $\Delta \mathcal{U}$ can be reformulated as $W \Delta \mathcal{U}(k) \leq w$ giving rise to the third inequality in (20b).

\section{MPC Implementation}

The tuning parameters of the MPC controller are the control and prediction horizons $H_{u}$ and $H_{p}$, along with the constraint matrices $E, F$ and $G$. This section discusses the choice of horizons and constraints. The $Q$ and $R$ weighting matrices will not be described as some trial and error is involved in their tuning.

The choice of prediction horizon is influenced by the time distance between two blades, in samples, at the nominal rotor speed, as the disturbance information from one blades is passed $H_{p}$ samples to the next. At the expected maximum rotor speed of $\omega_{\max }$ and a $2 \pi / 3$ distance between blades, the number of samples between two adjacent blades gives the prediction horizon,

$$
H_{p}=\frac{2 \pi / 3}{\omega_{\max } T_{s}} .
$$

For the wind turbine example $\omega_{\max }=2.32 \mathrm{rad} / \mathrm{s}$ with $H_{p} \approx$ 90. The control signal of the final value of $H_{u}$ must stabilize the model over the prediction horizon $H_{p}$. Choosing a small $H_{u}$ may result in fluctuating control signals, opposite to a larger one that results in a smoother actuation. By empirical tests $H_{u}=10$ has been chosen.

Constraints were applied to control inputs and their rates of change, and states, according to (20b). The generator torque and pitch angles were constrained to $\pm 20 \%$ and \pm 26 deg from their operating point values, respectively. Pitch rate limit were set to $\pm 10 \mathrm{deg} / \mathrm{s}$. State constraints help to enforce system stability and also guarantees that structural limits are not surpassed. Constraints were set on the three blade absolute positions to avoid the blade hitting the tower. The blades are allowed to move at $6 \mathrm{~m} / \mathrm{s}$ velocities. Tower position and tower velocity limits are set to $\pm 0.5 \mathrm{~m}$ and $\pm 0.05 \mathrm{~m} / \mathrm{s}$ respectively. The low speed shaft constraints are set to $\pm 1 \mathrm{rad} / \mathrm{s}$ to allow variable speed operation, and the high speed shaft to a factor of 100 larger due to the gear ratio.

\section{Simulation Results}

The proposed repetitive MPC controller is evaluated by non-linear simulation of a $1.5 \mathrm{MW}$ wind turbine. MPC performance is compared to a reference controller similar to the controller of the aeroelastic code FAST. One PID controller regulates rotational speed $\left(\omega_{H S S}\right)$ while another PID adds damping to the tower fore-aft motion by measuring the nacelle acceleration $\left(\ddot{x}_{\text {tower }}\right)$. Tuned through successive simulations to match the performance of the FAST controller, the PID gains are $K_{P}=-0.2, K_{I}=-0.8, K_{D}=0$ for the speed feedback and $K_{P}=0.1, K_{I}=2, K_{D}=0.2$ for the tower acceleration feedback.

The effectiveness of the proposed controller is illustrated by time series and FFT spectrum plots, as well as equivalent damage load of blades, tower, and low speed shaft obtained through a standard rainflow counting algorithm. The wind used during all tests is a $200 \mathrm{sec}$ time series of the stochastic wind field described in the modeling section. A slow sinusoidal signal has been added to the mean wind in order to ensure a mean wind deviation between 13 to $17 \mathrm{~m} / \mathrm{s}$.

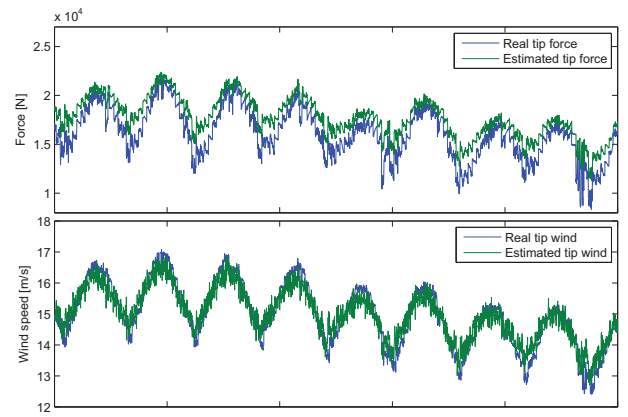

(a) Blade tip.

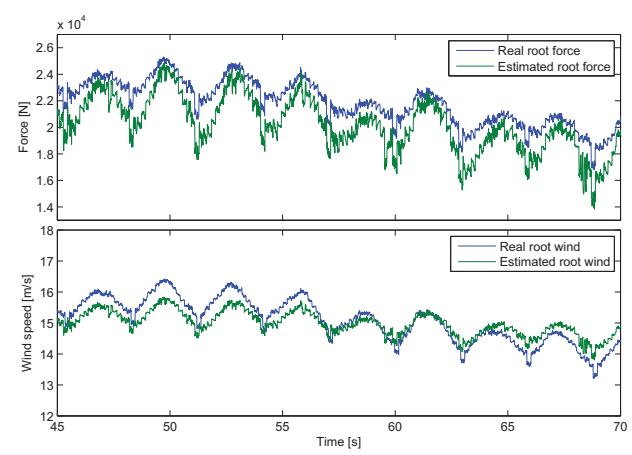

(b) Blade root.

Fig. 7. Comparison of real and estimated forces and winds. 
Figures 7(a) and 7(b) show forces and wind estimates of tip and root, respectively. The estimated tip wind has a very good agreement with the exact wind, although lower amplitudes can be noticed. The slightly negative offset and larger amplitude changes of the root force estimate can be explained by the influence of the strain gauge measuring the net axial force of the blade. Because the estimated forces generate inputs to the kinematic model, the estimated tip force is corrected by the blade velocity measurements. An error in the estimated tip velocity and position results in an distorted estimate of the tip force. The tip force error is corrected by the net axial force given by the strain gauge, causing an opposite sign error in the root force.

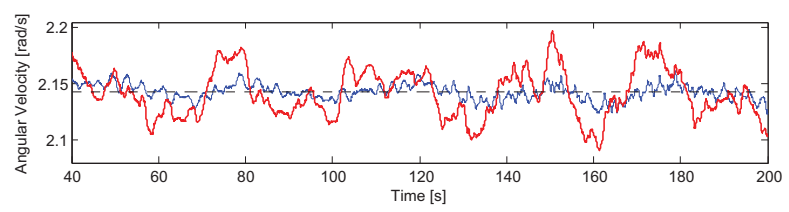

(a) Low speed shaft angular velocity.
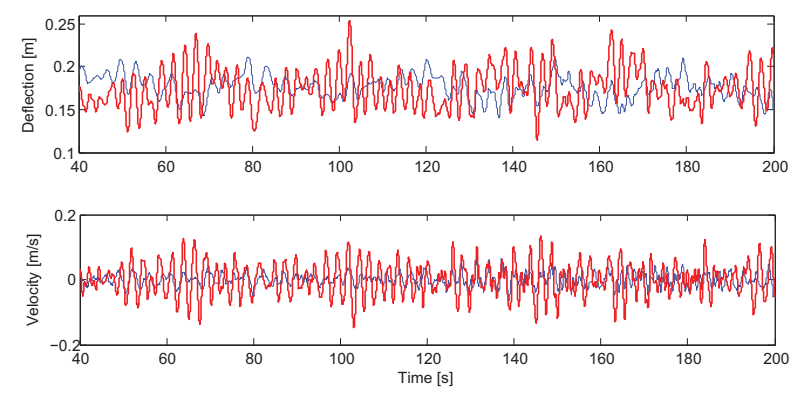

(b) Tower deflection and velocity.
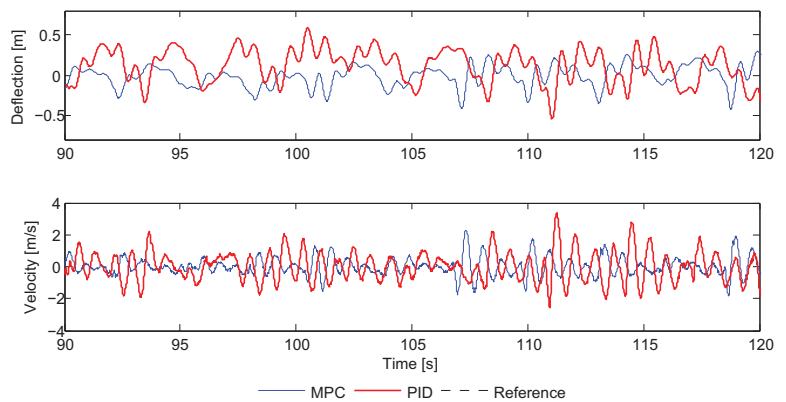

(c) Blade deflection and velocity.

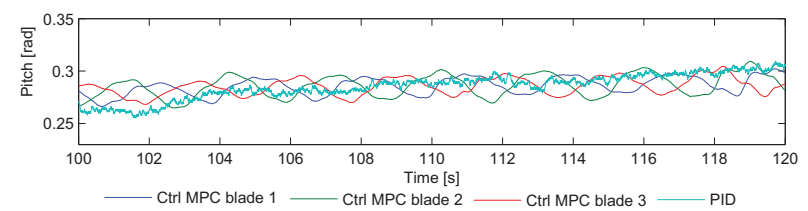

(d) Individual pitch angles.

Fig. 8. Time series results.

The times series plot of the blades pitch angle (Fig. 8(d)) clearly shows that the repetitive MPC controller generates an individual pitch like control signal. Time series of the low shaft angular velocity are depicted in Fig. 8(a). Tower deflections and velocities are depicted in Fig. 8(b). By realizing that smaller amplitudes occur on both signals, it is clear that a greater tower damping is proportioned by the MPC controller. Blade velocity is significantly slower in the MPC case as can be seen in Fig. 8(c). Blade deflections have similar amplitudes as the PID case.

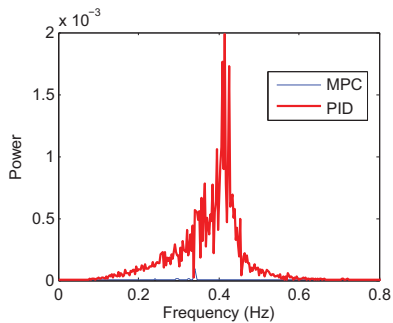

(a) Tower velocity.

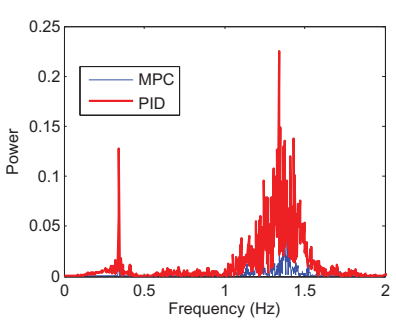

(b) Blade velocity.
Fig. 9. FFT results.

Attenuation of tower vibrations in a wide frequency range can be noticed from the FFT plot of tower velocity (Fig. 9(a)). The $1 \mathrm{P}$ frequency $(0.34 \mathrm{~Hz})$ has some small residual power. A FFT-analysis of the blade velocity shows that the repetitive MPC strategy with estimated wind disturbance mitigates blade vibrations in a wide frequency spectrum (Fig. 9(b)). The vibrations caused by $1 \mathrm{P}$ effects at $0.34 \mathrm{~Hz}$ are reduced significantly. This is also the case for the $4 \mathrm{P}$ content $(1.36 \mathrm{~Hz})$. The $4 \mathrm{P}$ frequency mainly originates from the wake dynamics of the wind field [12].

Equivalent loads were calculated for the MPC and PID controllers using a rainflow counting algorithm based on the standard [13]. When compared to the PID reference controller, low speed shaft decreased by a factor 6.8 , blades stress decreased by a factor 3.3 , and tower stress decreased by a factor 6.5 .

\section{REFERENCES}

[1] Bossanyi, E., Wind Turbine Control for Load Reduction. Wind Energy, Vol. 6, pg. 229-244, 2003.

[2] Bossanyi, E., Individual Blade Pitch Control for Load Reduction. Wind Energy, Vol. 6, pg. 119-128, 2003.

[3] Bossanyi, E. Controller field tests on the NREL CART2 turbine. UpWind Project Report 11593/BR/08, 2010, available at www.upwind.eu.

[4] Henriksen, L.C. Model Predictive Control of a Wind Turbine. Master Thesis, Technical University of Denmark, 2007.

[5] Korber, A.; King, R. Model Predictive Control for Wind Turbines. Proc. European Wind Energy Conference, Warsaw, 2010.

[6] Kumar, A.; Stol, K. Scheduled Model Predictive Control of a Wind Turbine, Proc. AIAA/ASME Wind Energy Symp., Jan. 2009.

[7] Friis, J.; Nielsen, E.; Bonding, J. Repetitive Individual Pitch Model Predictive Control for Horizontal Axis Wind Turbine. Master thesis, Aalborg University, 2010.

[8] D. S. Dolan and P. Lehn, Simulation model of wind turbine 3p torque oscillations due to wind shear and tower shadow, IEEE Trans. Energy Conversion, vol. 21, No. 3, 2006.

[9] Rehman, S. and Al-Abbadi, N. M., Wind shear coefficients and their effect on energy production, Energy Conversion and Management, Vol. 46, pg. 25782591, 2005.

[10] NWTC Design Codes (FAST by Jason Jonkman, Ph.D.). http://wind.nrel.gov/designcodes/simulators/fast/.

[11] Maciejowski, J. M. Predictive Control with Constraints. Prentice Hall, 2002.

[12] P. V. lund, Loads on a horizontal axis wind turbine operating in wake, J. of Wind Eng. and Industr. Aerodyn., vol. 39, pg. 317-328, 1992.

[13] ASTM E1049 - 85(2005) Standard Practices for Cycle Counting in Fatigue Analysis. 